\title{
P-Fuzzy Diffusion Equation Using Rules Base
}

\author{
Jefferson Leite, ${ }^{1}$ R. C. Bassanezi, ${ }^{2}$ Jackellyne Leite, ${ }^{3}$ and Moiseis Cecconello ${ }^{4}$ \\ ${ }^{1}$ Federal University of Piauí, 64063-040 Teresina, PI, Brazil \\ ${ }^{2}$ Federal University of ABC, 09210-170 Santo André, SP, Brazil \\ ${ }^{3}$ Federal Institute of Maranhão, 65635-468 Timon, MA, Brazil \\ ${ }^{4}$ Federal University of Mato Grosso, 78075-202 Cuiabá, MT, Brazil \\ Correspondence should be addressed to Jefferson Leite; jleite@ufpi.edu.br
}

Received 12 February 2014; Accepted 23 April 2014; Published 6 July 2014

Academic Editor: Laurent Gosse

Copyright (C) 2014 Jefferson Leite et al. This is an open access article distributed under the Creative Commons Attribution License, which permits unrestricted use, distribution, and reproduction in any medium, provided the original work is properly cited.

We propose a fuzzy system that simulates dispersion of individuals whose movements are described by diffusion. We will use only the position of the population as an input variable for describing the process. We emphasize that the classical diffusion equation along with its analytical solution in no time was used for obtaining our solution.

\section{Introduction}

The differential equations and deterministic differences are a powerful tool for modeling phenomena whose state variables are subject to changes over time. However, for the deterministic modeling be efficient is necessary to have a deep well of the relationship between the variables and their variations knowledge. It is the knowledge of the phenomenon that makes it possible to choose the functions that determine the variations with respect to the state (value) of the variable. In many cases, however, this relationship between variables and variations is only partially known, which make the deterministic model is less applicable [1-5].

On the other hand, models of fuzzy variational equations though behaving subjectivities are also not applicable modeling phenomena with partially known relationships. This comes from the fact that these models are derived from deterministic models. Subjectivity supported by fuzzy equations refers to uncertainties as the initial states of fuzziness demographic variables and parameters of fuzziness environmental. In general, both types of fuzziness are present in equations of population dynamics [6-9].

The p-fuzzy systems incorporate subjective information in both variables as the variations and their relationships with the variables and are therefore a very useful tool for modeling phenomena whose behavior is partially known.
The fuzzy systems are generally the result of a generalization of the classical systems; that is, in this approach the uncertain concepts are incorporated into these systems. A central feature of fuzzy systems is that they are based on the concept of fuzzy partition information. The use of fuzzy sets allows a generalization of information that is associated with the introduction of imprecision ignoring the phenomena. In essence, the representation of information in fuzzy systems tries to imitate the process of human reasoning, considering heuristic knowledge and information across the disconnected principle [10].

In this work, we describe a diffusive process without the use of their analytical solution, using dynamical systems p-fuzzy and given a rule base. It is worth noting that the results obtained in terms of solution are very similar to the deterministic.

\section{Preliminaries}

A subset (classic) $A$ of the universe $U$ can be represented by its characteristic function given by

$$
\chi_{A}(x)= \begin{cases}1, & \text { if } x \in A \\ 0, & \text { if } x \neq A .\end{cases}
$$




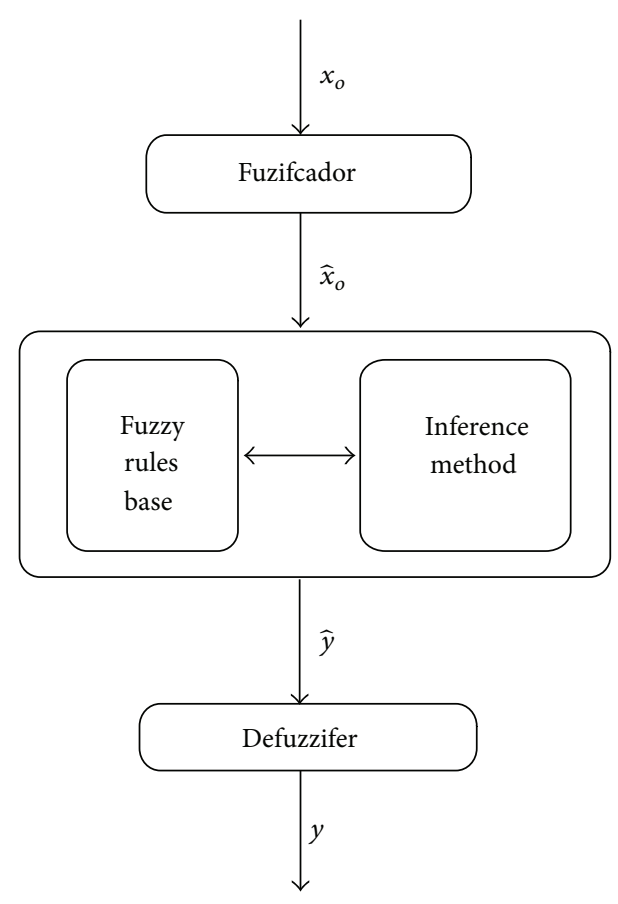

FIGURE 1: Structure of the fuzzy controller.

Thus, the membership function describes completely the set $A$, since it indicates which elements of the set $U$ are elements of $A$.

Allowing a kind of relaxation in the image set of the membership function of a set, Zadeh mathematically formulated the concept of fuzzy set [11]. We define a fuzzy subset $A$ of $U$, or simply fuzzy set, through the membership function $\mu_{A}: U \rightarrow[0,1]$, where $\mu_{A}(x)$ represents the degree of membership of the element $x$ to fuzzy subset $A$.

A fuzzy rule-based system has basically four components: an input processor (or fuzzificator), a set of linguistic rules, a model of fuzzy inference, and a processor output (or defuzzificator), generating a real number as output. Figure 1 illustrates the controller fuzzy.

The fuzzification is the process by which the input values of the system are converted to fuzzy sets with respective ranges of values where they are defined. It is a mapping of the field of real numbers to fuzzy field.

We can define the fuzzy roles by structures of the form If $\{$ background Then resulting\} used to describe specific situations that can be subjected to analysis a panel of experts, whose inference leads to a desired result. The background to define a fuzzy region in the space of input variables describes a system and condition, whereas the result define a region in the space of output variables of the system and describe a conclusion or an action that can be drawn when the premises occur. A fuzzy rule is a unit able to capture some specific knowledge.

A set of rules (or rule base) can describe a system in its various possibilities, fulfilling the role of translating mathematically the information of basis of knowledge of the fuzzy system. The rule base systems fuzzy (RBSF), in this case called fuzzy controllers, has four modules: the fuzzification module, the module based on linguistic rules, fuzzy inference,

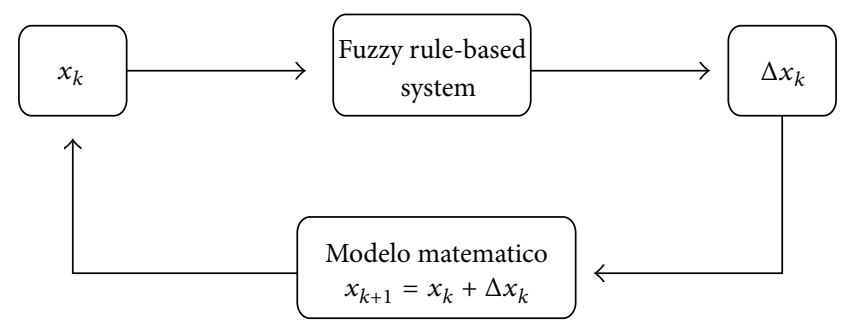

FIgURE 2: Structure of operation of a p-fuzzy system.

and defuzzification module. These modules are connected as shown in Figure 2. We call system $p$-fuzzy iterative system

$$
\begin{aligned}
& x_{k+1}=f\left(x_{k}\right), \\
& x_{o} \in \mathbb{R}^{n} \text { given, }
\end{aligned}
$$

where $f\left(x_{k}\right)$ is almost linear; that is, $f\left(x_{k}\right)=x_{k}+\Delta\left(x_{k}\right)$, $\Delta\left(x_{k}\right) \in \mathbb{R}^{n}$ and $\Delta\left(x_{k}\right)$ is obtained by a system based on fuzzy rules.

The rule base is a set consisting of fuzzy rules that relates the linguistic terms of the input variables and output variables. The rule base is considered as an element of a member of the fuzzy controller core. Each rule base satisfies the following structure:

$$
\text { IF } a \text { is in } A_{i} \text { THEN } b \text { is in } B_{i} \text {, }
$$

where $A_{i}$ and $B_{i}$ are fuzzy sets that represent linguistics terms of input variables and output variables, respectively. The expression $a$ in $A_{i}$ means $\mu_{A_{i}}(a) \in[0,1]$. Both the fuzzy set $A_{i}$ and $B_{i}$ can be a cartesian product of fuzzy sets; that is, $A_{i}=A_{i 1} \times A_{i 2} \times \cdots \times A_{i m}$ and $B_{i}=B_{i 1} \times B_{i 2} \times \cdots \times B_{i n}$. In this case, each fuzzy set, $A_{i j}$ and $B_{i k}$, is a linguistic term for the $j$ th input variable and $k$-th output variable, and the expression $a$ in $a_{i}$ means that

$$
\mu_{A_{i}}(a)=\min \left\{\mu_{A_{i 1}}, \mu_{A_{i 2}}, \ldots, \mu_{A_{i m}}\right\} \in[0,1] .
$$

It is the definition of the rule base the information of the phenomenon under study are used. For each state defined by the linguistic terms of the input variable is a rule base. Thus, the more linguistic terms more details are incorporated in the model.

The relationship between the linguistic variable is characterized MIN by the operator; that is, each rule is considered a fuzzy relation $R_{i}$ where the degree of membership for each $(a, b)$ is

$$
\mu_{R_{i}}(a, b)=\min \left\{\mu_{A_{i}}(a), \mu_{B_{i}}(b)\right\} .
$$

The relationship between each rule is characterized by the maximum operator, that is, fuzzy relation $R$, which is determined by the rule base model is obtained by joining each individual rule, so that for each pair $(a, b)$ we have

$$
\mu_{R}(a, b)=\max _{1 \leq i \leq n}\left\{\mu_{A_{i}}(a) \wedge \mu_{B_{i}}(b)\right\}
$$

where $\wedge$ represents the operator MIN. 


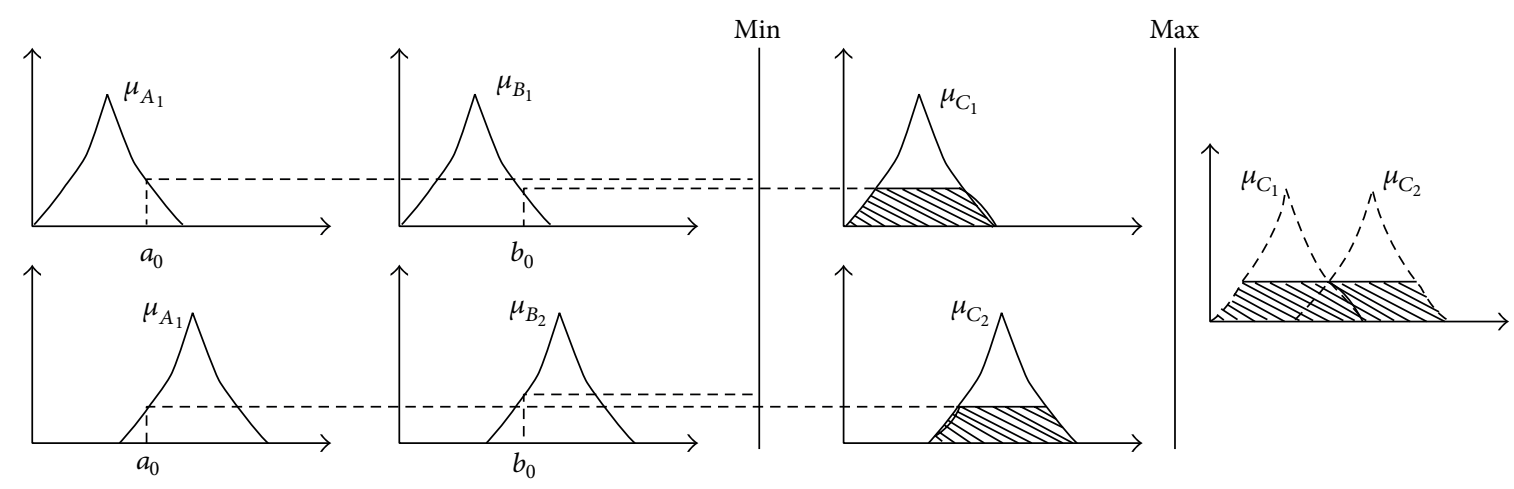

FIgURE 3: Mamdani inference engine with two linguistic input variables and one output.

Now we want to find each entry a corresponding action; that is, for a $A$ set of input data, we determine a set $B$ output data. Mamdani method, the membership function of $B$, is given by

$$
\mu_{B}(b)=\max _{1 \leq i \leq n}\left\{\max _{a}\left\{\mu_{A}(a) \wedge \mu_{A_{i}}(a)\right\} \wedge \mu_{B_{i}}(b)\right\} .
$$

If the entry is a classic unitary set, then $\mu_{A}(a)=1$ and $\mu_{A_{i}}(a) \leq 1$. Therefore, the previous expression results in

$$
\mu_{B}(b)=\max _{1 \leq i \leq n}\left\{\mu_{A_{i}}(a) \wedge \mu_{B_{i}}(b)\right\}
$$

and therefore we have the fuzzy set $B$ representing the action of each input $A$ (Figure 3 ).

The role of the defuzzificador is to convert each completion obtained by the method of inference in a real number which is the best action to take. For systems p-fuzzy, the actual number is obtained by defuzzification added to the value assumed by input variable in instant $k$, feeding the iterative system.

One of the main methods of defuzzification is the center of mass, for continuous variables, which is given by

$$
m(B)=\frac{\int_{\Omega} b \mu_{B}(b) d b}{\int_{\Omega} \mu_{B}(b) d b} .
$$

This defuzzification method will be used throughout this paper. Note that the fuzzy controller can be seen as a function $f: \mathbb{R}^{n} \rightarrow \mathbb{R}^{m}$, since given an input value, there is a single output value.

\section{The Model}

In this section, we are interested in developing a base of rules that enable us to find a solution to a graphical problem involving diffusion. For this, we use only the position population, initial condition, and population growth. By these ways we may estimate the population density in an instant $t=$ $t^{*}$ without necessarily using the analytical solution problem.

Classical models of population dynamics and/or epidemiology, in overall, are given by a system of differential equations. In this case, the parameters of the models are often taken as mean values obtained from one set of data such

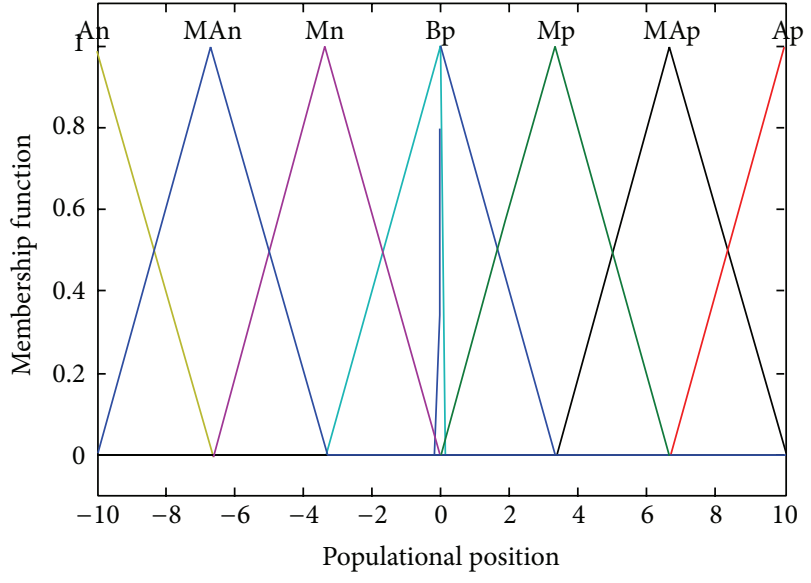

FIGURE 4: Linguistic variables for population position.

that the model is to be deterministically known. However, admitting uncertainty due to partial knowledge, which is common in biological phenomena, an alternative is to model such knowledge from a set of rules of the form if-then.

It is common to adopt an equation

$$
\frac{d y}{d t}=f(y)
$$

to represent the dynamic system, where the field $f$ is variation, from which the evolution of the system is studied. Actually we can ask the following question: how can we analyze system (10) if it is partially known? The response is to adopt a linguistic model capable of capable of capturing the information availables from the model with a specialist. Propose a methodology to estimate solutions to differential equations using fuzzy controllers in which the state variables are the inputs and outputs are changes of state.

Thus, consider how linguistic variables to position of the population (distance to origin): low positive (Bp), mean positive (Mp), mean high positive (MAp), positive high (Ap), low negative $(\mathrm{Bn})$, mean negative $(\mathrm{Mn})$, mean high negative (MAn) and negative high (An). Where the positive or negative terms means distance from the origin to the right or left respectively. Thus, Figure 4 represents graphically these linguistic variables. 


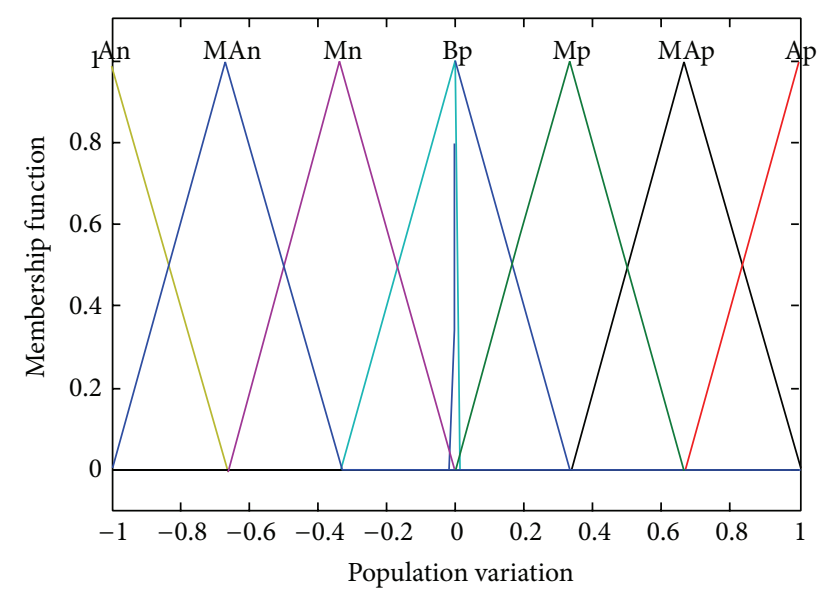

FIGURE 5: Linguistic variables for population growth.

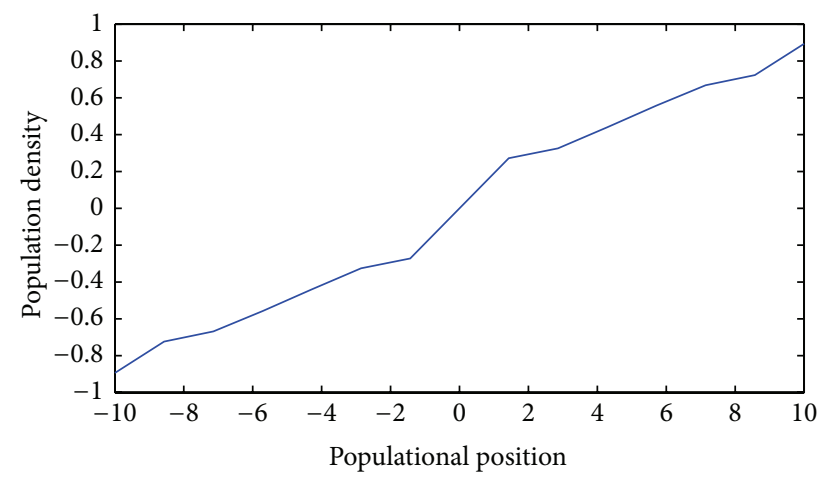

Figure 6: Curve generated from the rules of the previous figure using Mamdani.

Likewise, as output variable, consider the variation population and linguistic variables: low positive (Bp), positive average (Mp), average high positive (MAp), high positive (Ap), low negative $(\mathrm{Bn})$, average negative $(\mathrm{Mn})$, average high negative (MAn), and high negative (An). In Figure 5 these plots represent the linguistic variables.

Considering the known results about diffusion process, consider the following of fuzzy rules:

(a) if the position is of the individuals low positive Bp then the variation of population is low positive $\mathrm{Bp}$;

(b) if the position is of the individuals positive average $\mathrm{Mp}$ then the variation of population is positive average Mp;

(c) if the position is of the individuals average high positive MAp then the variation of population is average high positive MAp;

(d) if the position is of the individuals high positive Ap then the variation of population is high positive Ap;

(e) if the position is of the individuals low negative $\mathrm{Bn}$ then the variation of population is low negative $\mathrm{Bn}$;

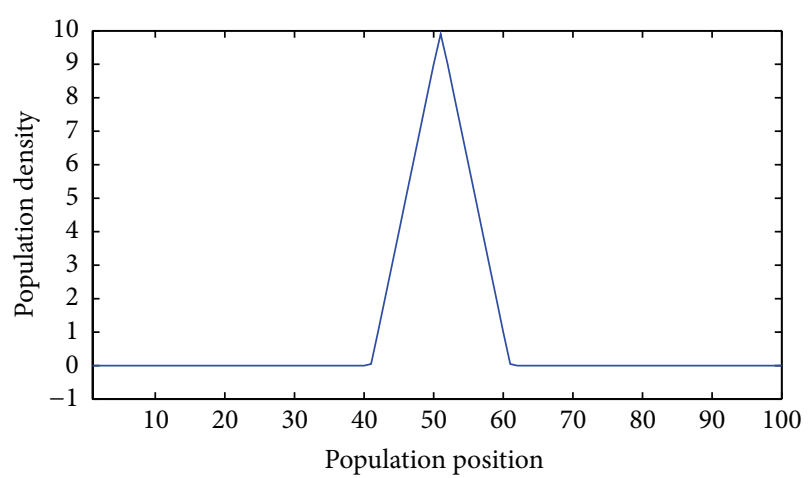

FIgURE 7: Solution to $t=0$.

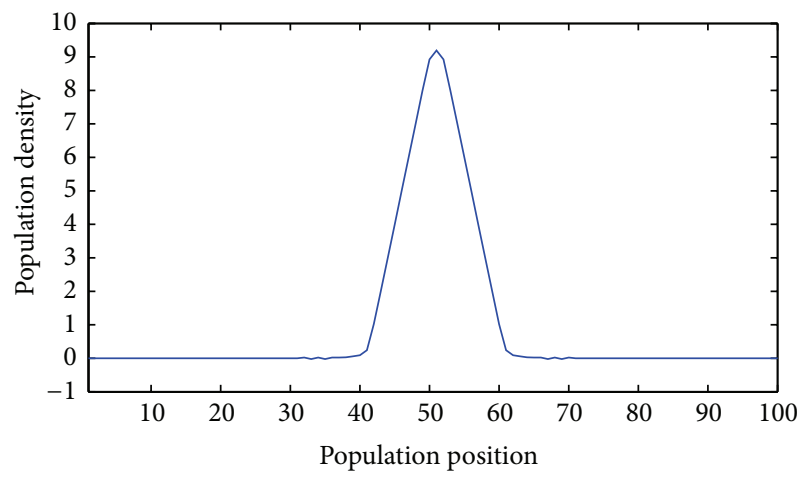

FIGURE 8: Solution to $t=10$.

(f) if the position is of the individuals average negative $\mathrm{Mn}$ then the variation of population is average negative $\mathrm{Mn}$;

(g) if the position is of the individuals average high negative MAn then the variation of population is average high negative MAn;

(h) if the position is of the individuals high negative An then the variation of population is high negative An.

Figure 6 shows the graph of all variation of dependent variable (change in population) as a function of independent variable (position of the population) in the Mamdani model.

So with this rule base, the Mamdani controller and defuzzification given by the center of mass, the p-fuzzy system in leads to the path illustrated by the sequence of Figures $7,8,9,10,11,12,13$, and 14 . To this end, we consider as a condition initial $N_{0}=10$.

Another important observation is that time in our problem means the number of iterations in simulated MATLAB. This information is of great importance because it reduces the amount of data needed to describe the problem. We can thus write $\mathrm{p}$-fuzzy dynamical system in the following form:

$$
x_{k+1}=x_{k}+\Delta x_{k} .
$$

As for each iteration $\left(t=t^{*}\right)$, we have a curve; then, we can plot a surface that will be our graph p-fuzzy solution of the diffusion equation. This graph is shown in Figure 15. 


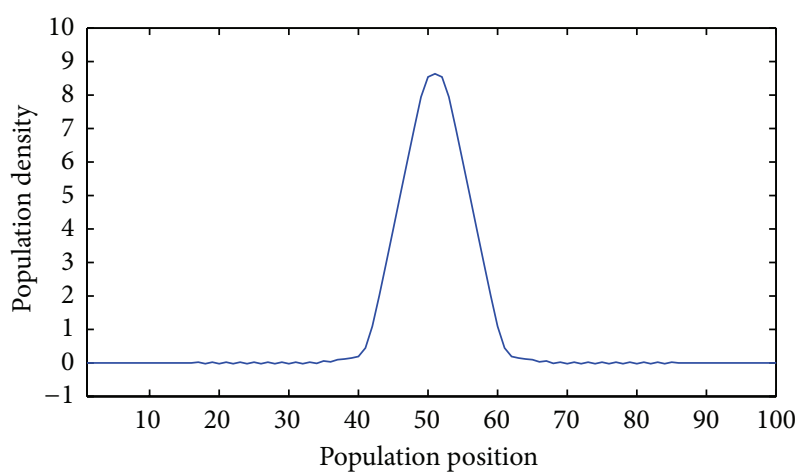

FIGURE 9: Solution to $t=25$.

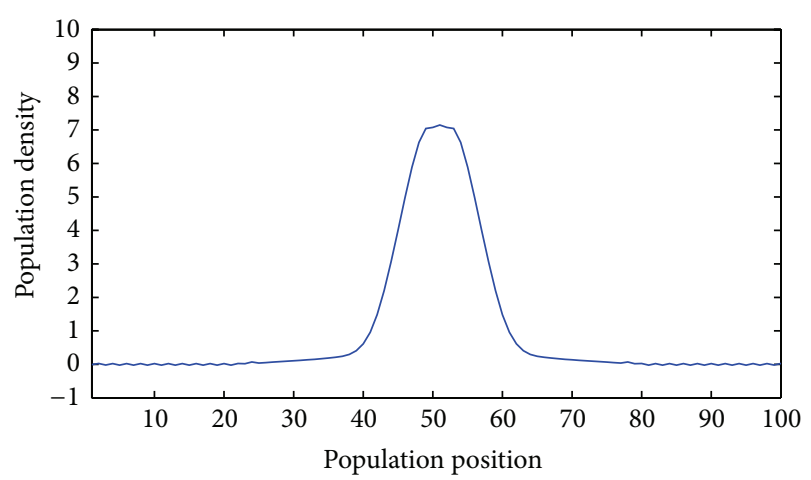

FIGURE 10: Solution to $t=100$.

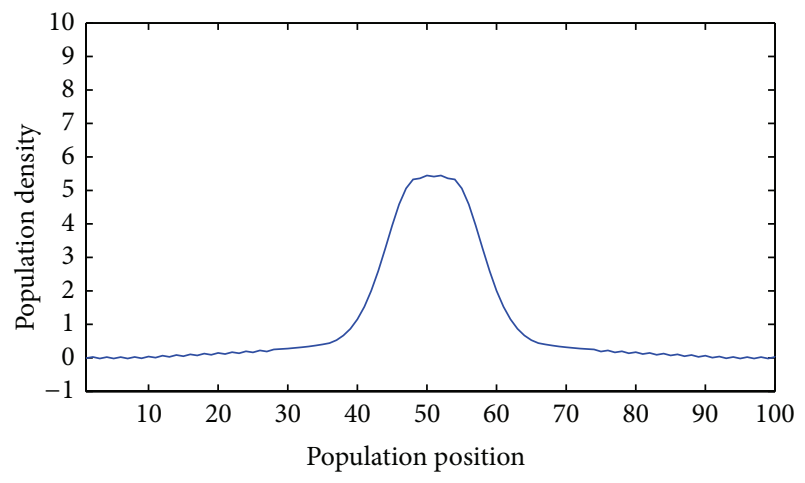

FIgURE 11: Solution to $t=250$.

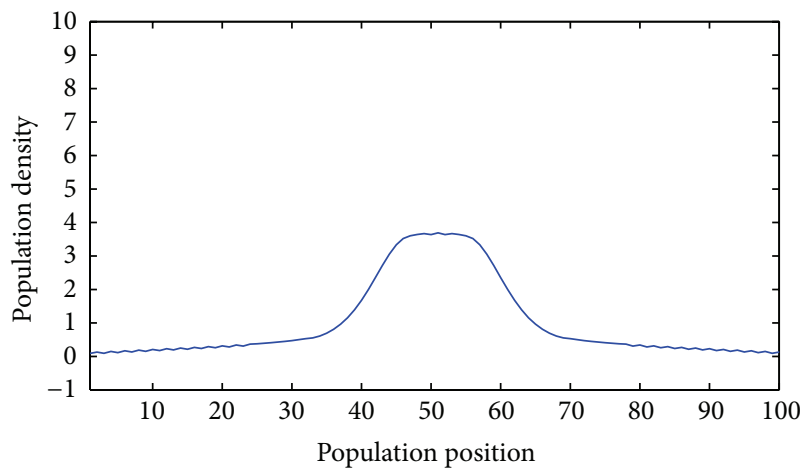

FIGURE 12: Solution to $t=500$.

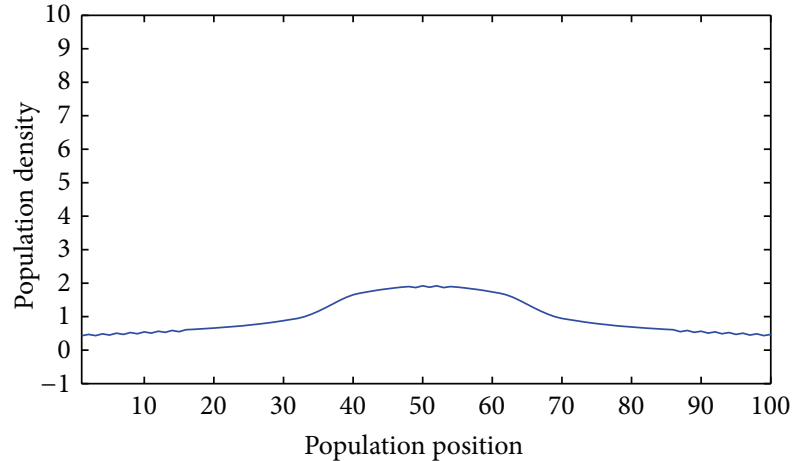

Figure 13: Solution to $t=1000$.

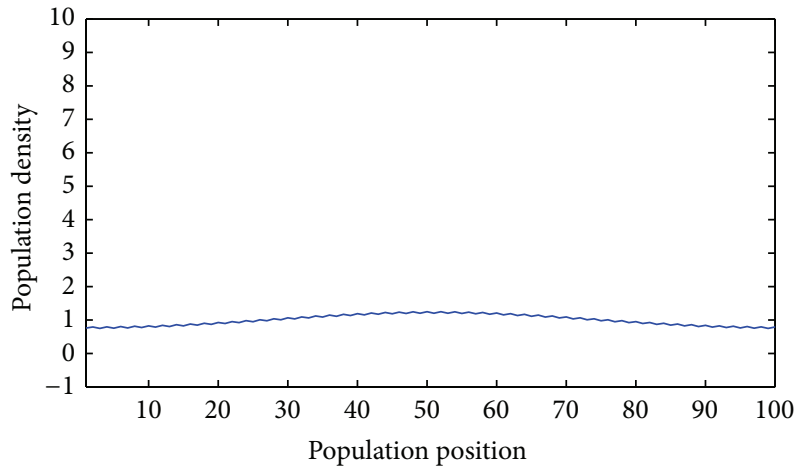

FIGURE 14: Solution to $t=5000$.

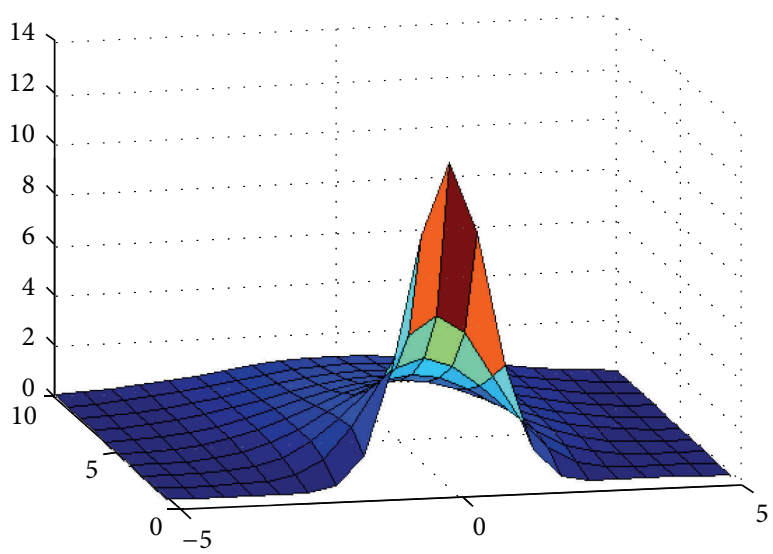

FIGURE 15: Union of p-fuzzy solutions.

\section{Conclusion}

The most interesting in this process is not possible to know what the best model, it is the deterministic model or the pfuzzy model, since the results are very similar as we can see in Figure 15. The modeling assumes that not alwayshas real data, the intuition or common sense can guide the formulation of models.

From the educational point of view the best model is secondary because you can always do better than the previous one and you can always imagine different situations for the same phenomenon. 
We show that it is possible to use a FRBS to model the behavior of the population density of a species when you want to take into account the diffusion of individuals.

\section{Conflict of Interests}

The authors declare that there is no conflict of interests regarding the publication of this paper.

\section{References}

[1] P. Diamond, "Stability and periodicity in fuzzy differential equations," IEEE Transactions on Fuzzy Systems, vol. 8, no. 5, pp. 583-590, 2000.

[2] P. Diamond, "Brief note on the variation of constants formula for fuzzy differential equations," Fuzzy Sets and Systems, vol. 129, no. 1, pp. 65-71, 2002.

[3] A. Kandel and W. J. Byatt, "Fuzzy differential equations," in Proceedings of the International Conference on Cybernetics and Society, Tokyo, Japan, 1981.

[4] M. T. Malinowski, "On random fuzzy differential equations," Fuzzy Sets and Systems, vol. 160, no. 21, pp. 3152-3165, 2009.

[5] J. D. Murray, Mathematical Biology I. An Introduction, vol. 1, Springer, New York, NY, USA, 2002.

[6] M. S. Cecconello, R. C. Bassanezi, A. V. Brandão, and J. Leite, "Periodic orbits for fuzzy flows," Fuzzy Sets and Systems, vol. 230, pp. 21-38, 2013.

[7] M. S. Cecconello, J. Leite, R. C. Bassanezi, J. de Deus, and M. Silva, "About projections of solutions for fuzzy differential equations," Journal of Applied Mathematics, vol. 2013, Article ID 184950, 9 pages, 2013.

[8] J. D. Silva, J. Leite, R. C. Bassanezi, and M. S. Cecconello, "Stationary points-I: one-dimensional p-fuzzy dynamical systems," Journal of Applied Mathematics, vol. 2013, Article ID 495864, 11 pages, 2013.

[9] M. T. Mizukoshi, L. C. Barros, and R. C. Bassanezi, "Stability of fuzzy dynamic systems," International Journal of Uncertainty, Fuzziness and Knowledge-Based Systems, vol. 17, no. 1, pp. 69-83, 2009.

[10] J. Leite, Sistemas dinâmicos fuzzy aplicado a processos difusivos [Ph.D. thesis], Universidade Estadual de Campinas, Campinas, Brazil.

[11] L. A. Zadeh, "Fuzzy sets," Information and Control, vol. 8, pp. 338-353, 1965. 


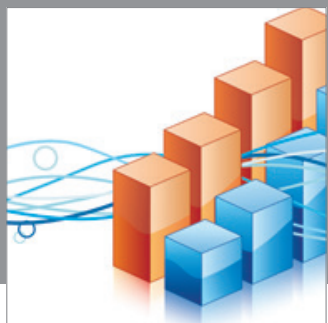

Advances in

Operations Research

mansans

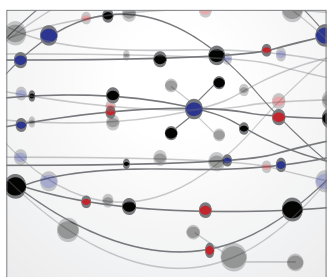

The Scientific World Journal
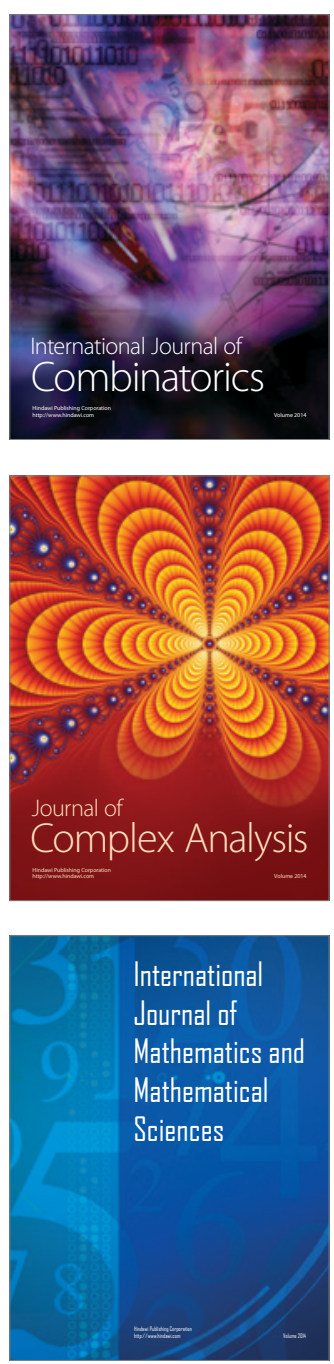
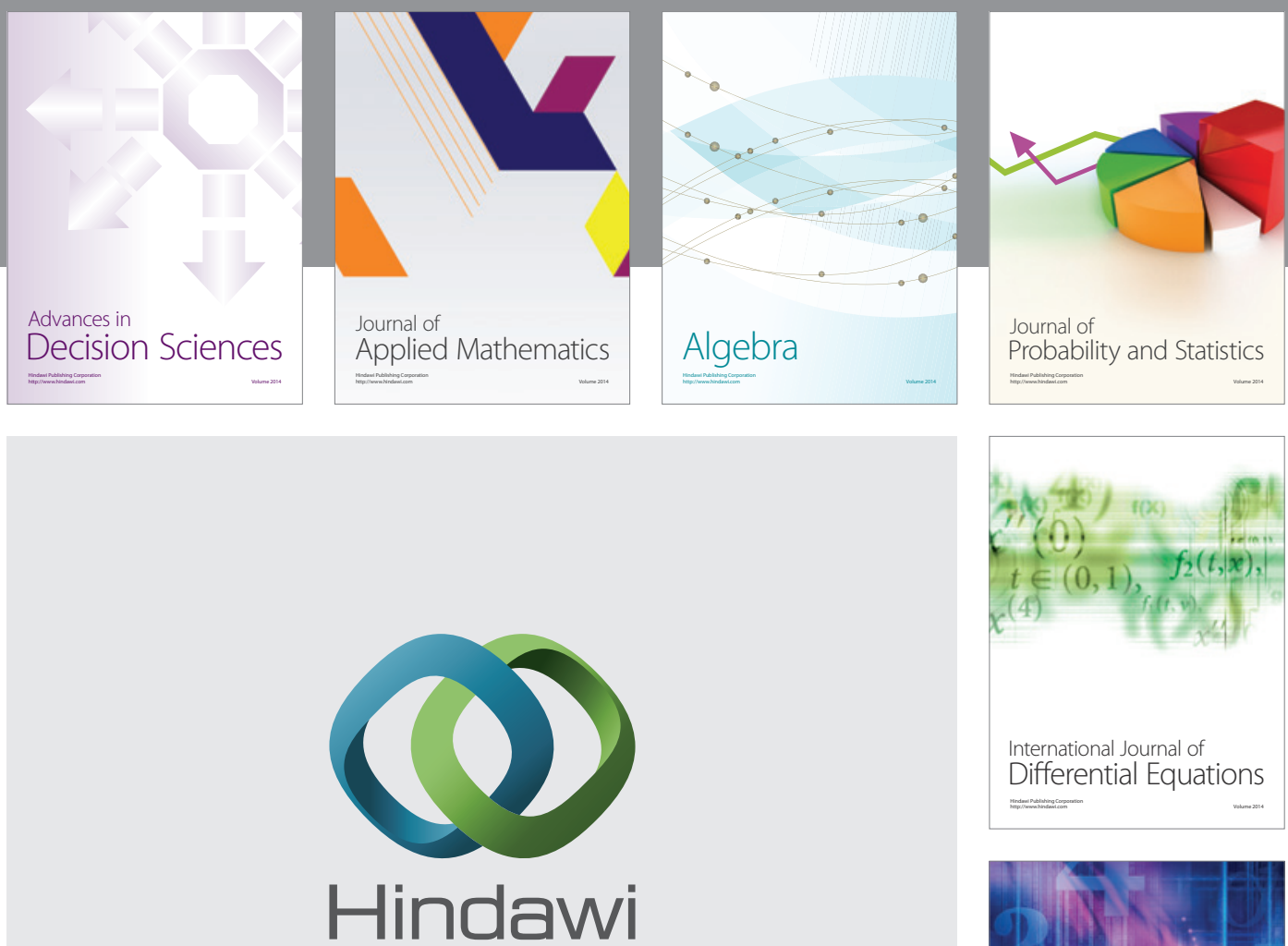

Submit your manuscripts at http://www.hindawi.com
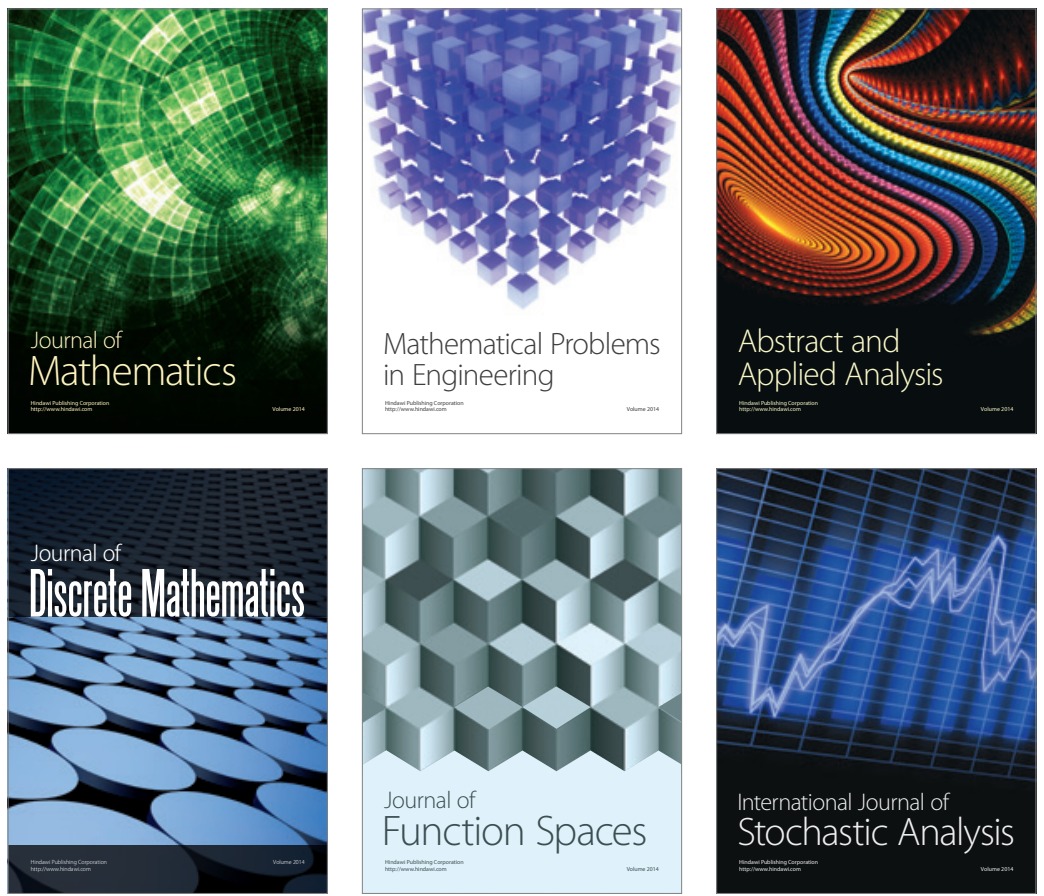

Journal of

Function Spaces

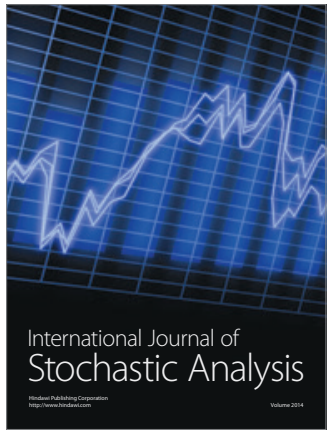

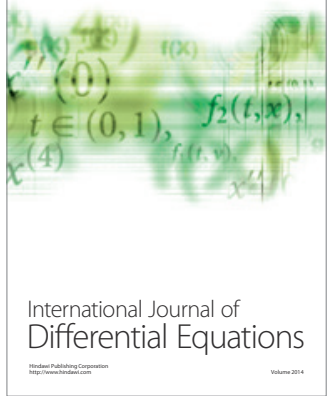
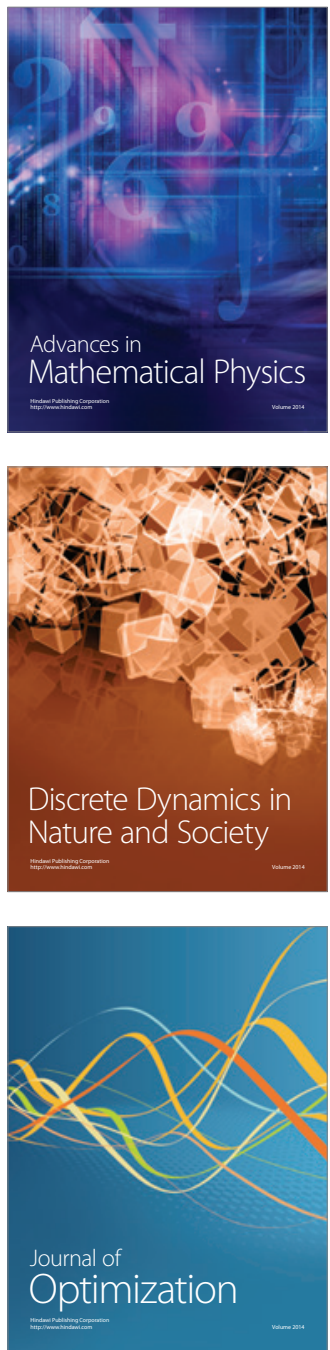